\title{
COMPACTAÇÃO DE SOLOS EM LABORATÓRIO: EFEITO DO DIÂMETRO E DO NÚMERO DE CAMADAS DO CORPO DE PROVA
}

Flavio Alessandro Crispim², Dario Cardoso de Lima $^{3}$, Claudio Henrique de Carvalho Silva ${ }^{3}$, Carlos Ernesto Gonçalves Reynaud Schaefer ${ }^{4}$, Carlos Alexandre Braz de Carvalho ${ }^{3}$ e Elisson Hage Brandão ${ }^{5}$

RESUMO - Neste trabalho, estudou-se a influência do diâmetro e do número de camadas do corpo de prova nas curvas de compactação de laboratório e na resistência mecânica de dois solos residuais de gnaisse da Zona da Mata Norte de Minas Gerais, respectivamente, de texturas argilo-areno-siltosa (Solo 1) e areno-silto-argilosa (Solo 2), com vistas à construção de estradas florestais. Além dos resultados dos ensaios de compactação, determinou-se a resistência à compressão não confinada dos solos empregando corpos de prova compactados na umidade ótima $\left(\mathrm{w}_{\mathrm{ot}}\right)$ e nos teores de umidade 3\% abaixo e $2 \%$ acima, considerando como referência a energia de compactação do ensaio Proctor normal e empregando corpos de prova compactados em uma, duas e três camadas, bem como nos diâmetros de $35 \mathrm{~mm}, 73 \mathrm{~mm}$ e $100 \mathrm{~mm}$, com nove repetições. Para fins práticos de engenharia e com base em análise estatística aplicada aos parâmetros massa específica aparente seca e resistência à compressão não confinada, pode-se concluir que: (i) há diferenças significativas entre as compactações realizadas em uma e em três camadas, não ocorrendo o mesmo nas compactações realizadas em duas e em três camadas, para ambos os solos; e (ii) há diferenças significativas entre a compactação de corpos de prova de diâmetro $100 \mathrm{~mm}$ e os demais de $73 \mathrm{~mm}$ e $35 \mathrm{~mm}$, para o solo 1 (argiloso), bem como há também diferenças apenas no ramo seco da curva de compactação, para o solo 2 (arenoso).

Palavras-chave: Compactação de solos; Resistência à compressão não confinada; Parâmetros de compactação.

\section{LABORATORY SOILS COMPACTION: EFFECT OF SPECIMEN DIAMETER AND NUMBER OF LAYERS}

\begin{abstract}
This paper addresses the study of the influence of specimen diameter and number of layers on the laboratory compaction curves and mechanical strength of two gneiss residual soils from Zona da Mata Norte of Minas Gerais state, Brazil, respectively with grain size distributions classified as silty-sandy clay (Soil 1) and clayey-silty sand (Soil 2) for forest road applications. Besides compaction testing data, it was determined the unconfined compression strength of soils using specimens compacted at the optimum water content $\left(w_{o}\right), w_{o t}-3 \%$ and $w_{o t}+2 \%$ referred to the standard Proctor compaction effort and using specimens compacted with one, two and three layers as well as diameters of $35 \mathrm{~mm}, 73 \mathrm{~mm}$ and $100 \mathrm{~mm}$, with nine replicates. For practical engineering purpose and based on statistical analysis applied to the parameters dry specific weight and unconfined compression strength it can be concluded that: (i) for both soils, there are significant differences between the compactions carried out in one and three layers, and not the same for two and three layers; and (ii) for Soil 1 (clayey soil), there are significant differences between compaction using $100 \mathrm{~mm}$ and the $73 \mathrm{~mm}$ and $35 \mathrm{~mm}$ diameter specimens, as well as for Soil 2 (sandy soil) these differences occurs only in the dry branch of the compaction curve.
\end{abstract}

Keywords: Soil compaction; Unconfined compression strength; Compaction parameters.

\footnotetext{
${ }^{1}$ Recebido em 11.09.2012 aceito para publicação em 24.03.2015.

${ }^{2}$ Universidade do Estado do Mato Grosso, Campus de Sinop, Faculdade de Ciências Exatas e Tecnológicas, Sinop, MT - Brasil. E-mail: <<crispim_br@yahoo.com.br>.

${ }^{3}$ Universidade Federal de Viçosa, Campus de Viçosa, Departamento de Engenharia Civil, Viçosa, MG - Brasil. E-mail: < declima@ufv.br>, <silvac@ufv.br>e <cabraz@ufv.br>.

${ }^{4}$ Universidade Federal de Viçosa, Campus de Viçosa, Departamento de Solos, Viçosa, MG - Brasil. E-mail: <carlos.schaefer@ufv.br>.

${ }^{5}$ Universidade Federal de Viçosa, Graduado em Engenharia Civil, Itabuna, BA - Brasil. E-mail: <declima@ufv.br>.
}

Revista Árvore, Viçosa-MG, v.39, n.3, p.535-542, 2015

http://dx.doi.org/10.1590/0100-67622015000300014 


\section{INTRODUÇÃO}

As estradas florestais brasileiras possuem extensão de aproximadamente $600.000 \mathrm{~km}$ (MACHADO; SILVA, 2001). Em geral, são estradas de terra em leito natural ou compostas por revestimento primário colocado sobre o subleito, sendo comumente projetadas e construídas sem critério racional de dimensionamento. Nesse contexto, é de suma importância a sua execução segundo as exigências das normas técnicas de outras obras viárias, dando-se a importância devida ao emprego das técnicas de compactação dos solos no campo e em laboratório. Com relação a trabalhos prévios realizados na Universidade Federal de Viçosa (UFV) sobre compactação de solos com direcionamento para estradas florestais, pode-se referir às abordagens de Machado et al. (2009) sobre a técnica de correção granulométrica aplicada a misturas solo-grits e de Silva et al. (2011) relativa à avaliação do subleito de estradas vicinais de baixo volume de tráfego e, especificamente, sobre o problema analisado neste artigo, referente às publicações de Crispim (2010) e Crispim et al. (2011; 2012).

Embora a compactação de campo seja a modalidade de maior interesse nos estudos de compactação de solos para fins viários, estudos desta natureza, via de regra, envolvem processos demorados e relativamente caros. Entretanto, a compactação em laboratório possibilita o controle adequado das condições de ensaio e melhor acurácia nas medições realizadas, além de permitir a simulação de situações que se assemelham àquelas de campo, sendo, em geral, a base para a definição dos parâmetros de compactação dos solos no campo.

Vários processos são utilizados para a compactação dos solos em laboratório, em que se podem variar o tipo de compactação, as energias de compactação empregadas, o número de camadas dos corpos de prova e as dimensões dos cilindros de compactação. Com relação aos dois últimos itens, a definição do número adequado de camadas relaciona-se com a busca da melhor distribuição do esforço de compactação ao longo do corpo de prova, buscando-se garantir a sua homogeneidade, ao passo que a definição do diâmetro do corpo de prova se relaciona, em regra, com as dimensões da maior partícula do solo a ser ensaiado. No último caso, pode-se referir, também, ao fato de que o emprego de corpos de prova de dimensões menores do que as padronizadas por norma técnica pode reduzir significativamente o volume de solo necessário para a realização de um estudo específico.
No Brasil, segundo o ensaio de compactação padronizado pela Associação Brasileira de Normas Técnicas - ABNT (ABNT, 1986), adota-se corpo de prova com três ou cinco camadas, enquanto o Departamento Nacional de Estradas de Rodagem (DNER), hoje Departamento Nacional de Infraestrutura de Transportes (DNIT), recomenda, também, o diâmetro de $50 \mathrm{~mm}$ (DNER, 1994); no entanto, na Metodologia Miniatura Compactada Tropical (MCT), por exemplo, há referência aos diâmetros de 50 mm e 26 mm (NOGAMI; VILLIBOR, 1995).

Neste trabalho, avaliou-se a influência do número de camadas e do diâmetro do corpo de prova no comportamento mecânico de dois solos tropicais da Zona da Mata Norte de Minas Gerais, Brasil, adotando como elementos de análise as curvas de compactação e a resistência à compressão não confinada dos solos e considerando a aplicação potencial em estradas florestais, via uso da energia do ensaio Proctor normal, mas também uso mais amplo no projeto e construção de rodovias.

\section{MATERIAL E MÉTODOS}

Este estudo foi realizado no Laboratório de Engenharia Civil do Departamento de Engenharia Civil da UFV, utilizando dois solos residuais de gnaisse da Zona da Mata Norte de Minas Gerais, Brasil, como se segue: (i) solo 1, residual maduro (horizonte B) de tonalidade amarelada, aspecto visual poroso e textura argilo-areno-siltosa. O local de coleta da amostra foi um talude de corte localizado no lado direito da Rodovia BR-120, que liga as cidades de Viçosa e Paula Cândido, próximo à Usina de Pré-Misturado a Frio da cidade de Viçosa, com coordenadas 2045'35"S e 4252'28"W; e (ii) solo 2, residual jovem (horizonte C), oriundo de um perfil de intemperismo de solos desenvolvidos de gnaisse do Pré-Cambriano de tonalidade acinzentada e textura areno-silto-argilosa. O local de coleta da amostra foi um talude de corte localizado na Vila Secundino, no Campus da UFV, com coordenadas $20^{\circ} 45^{\prime} 38^{\prime \prime S ~ e ~}$ $42^{\circ} 52^{\prime} 25^{\prime \prime W}$. Refere-se que os solos 1 e 2 são típicos do subleito de estradas na Zona da Mata Norte de Minas Gerais, considerando-se o lançamento de greide nos horizontes $\mathrm{B}$ e $\mathrm{C}$, respectivamente.

Os solos foram caracterizados geotecnicamente, de acordo com as recomendações da Associação Brasileira de Normas Técnicas - ABNT, sendo realizados os ensaios de análise granulométrica por peneiramento 
e sedimentação com o uso de hexametafosfato de sódio (ABNT, 1984a), limite de liquidez (ABNT, 1984b), limite de plasticidade (ABNT, 1984c) e massa específica dos sólidos (ABNT, 1984d). A escala granulométrica adotada para a caracterização das frações argila, silte, areia e pedregulho dos solos em análise foi a preconizada pela ABNT (1995).

Na busca de reproduzir condições de umidade próximas dos processos de compactação de solos no campo, com direcionamento a aterros, subleito e revestimentos primários de estradas florestais, adotaram-se a energia do ensaio de compactação Proctor normal (ABNT, 1986) e o critério de representar as curvas de compactação através de corpos de prova compactados na umidade ótima $\left(\mathrm{w}_{\mathrm{ot}}\right)$, umidade ótima menos $3 \%\left(\mathrm{w}_{\mathrm{ot}}-3 \%\right)$ e umidade ótima mais $2 \%$ $\left(\mathrm{w}_{\mathrm{ot}}+2 \%\right)$, trabalhando-se com nove repetições para cada teor de umidade. Os corpos de prova foram compactados após $24 \mathrm{~h}$ do umedecimento de cada amostra dos solos em estudo.

Considerando que nesta pesquisa se buscou analisar apenas o efeito do diâmetro e número de camadas nos parâmetros de compactação e de resistência mecânica dos solos, adotou-se a compactação estática como referência para a confecção dos corpos de prova, obtendo a respectiva energia de compactação do modo estático a partir do modo dinâmico tradicional, como se segue: (i) obter a curva de compactação dos solos utilizando a compactação dinâmica, segundo a ABNT (1986), ou seja, corpos de prova compactados em três camadas, utilizando cilindro de compactação Proctor de $100 \mathrm{~mm}$ de diâmetro e 127,3 mm de altura (aproximadamente, volume de $1.000 \mathrm{~cm}^{3}$ ), aplicando 27 golpes por camada do soquete de $2,5 \mathrm{~kg}$ e caindo de uma altura de 305 $\mathrm{mm}$. A partir da curva obtida, determinou-se a massa específica aparente seca $\left(\gamma_{s}\right)$ de cada teor de umidade (w) desejado; e (ii), a partir dos dados obtidos na etapa anterior, compactaram-se os solos estaticamente em uma, duas e três camadas, aplicando em cada camada a força necessária para se atingir o $\tilde{\mathrm{a}}_{\mathrm{s}}$ esperado, mediante o emprego de uma prensa hidráulica.

No estudo da influência do número de camadas de solo dos corpos de prova, utilizou-se um cilindro de compactação tripartido de dimensões de diâmetro $73 \mathrm{~mm}$ e altura de $150 \mathrm{~mm}$. Entretanto, no estudo da influência do diâmetro do cilindro de compactação, trabalhou-se com um conjunto de três cilindros, como se segue: (i) $100 \mathrm{~mm}$ de diâmetro e $120 \mathrm{~mm}$ de altura (cilindro Proctor); (ii) $73 \mathrm{~mm}$ de diâmetro e $150 \mathrm{~mm}$ de altura; e (iii) $35 \mathrm{~mm}$ de diâmetro e $80 \mathrm{~mm}$ de altura. Os resultados dos estudos referem-se a nove repetições.

Nos corpos de prova compactados, foram realizados ensaios de resistência à compressão não confinada (RCNC), segundo a NBR 12770/92 (ABNT 1992), sendo os corpos de prova ensaiados imediatamente após a compactação, à velocidade de $0,75 \mathrm{~mm} / \mathrm{min}$.

$\mathrm{Na}$ análise estatística dos resultados, empregaram-se os testes t e F a 5\% de probabilidade.

A Figura 1 apresenta um resumo das atividades realizadas nesta pesquisa, com descrição das etapas de coleta, processamento e realização dos ensaios de caracterização geotécnica das amostras de solos, definição do número de corpos de prova relativos a cada modalidade de estudo, referência aos ensaios de resistência à compressão não confinada, bem como dos estudos estatísticos.

\section{RESULTADOS}

Na Tabela 1, têm-se os resultados dos ensaios de caracterização e compactação realizados nos solos 1 e 2 .

Na Figura 2, apresentam-se os resultados dos ensaios de compactação (massa específica aparente seca $\left(\gamma_{\mathrm{s}}\right)$ versus teor de umidade $\left.(\mathrm{w})\right)$ e de resistência à compressão não confinada (resistência à compressão não confinada (RCNC) versus teor de umidade (w)) para os solos 1 e 2, considerando os estudos da influência do número de camadas dos corpos de prova (itens a e b) e do diâmetro do corpo de prova (itens c e d). Na Figura 3, ilustram-se as diferenças entre médias obtidas dos parâmetros $\gamma_{\mathrm{s}}$ e RCNC nesses solos, considerando como referência, respectivamente, a compactação em três camadas (itens a e b) e o diâmetro de $100 \mathrm{~mm}$ (itens c e d).

Na Tabela 2, apresentam-se os resultados da análise de variância aplicada aos dados obtidos nos solos 1 e 2 para o parâmetro RCNC, considerando os estudos da influência do número de camadas e do diâmetro do corpo de prova, para um nível de probabilidade de $5 \%$.

\section{DISCUSSÃO}

Considerando os resultados dos ensaios de caracterização apresentados na Tabela 1 , tem-se que

Revista Árvore, Viçosa-MG, v.39, n.3, p.535-542, 2015 


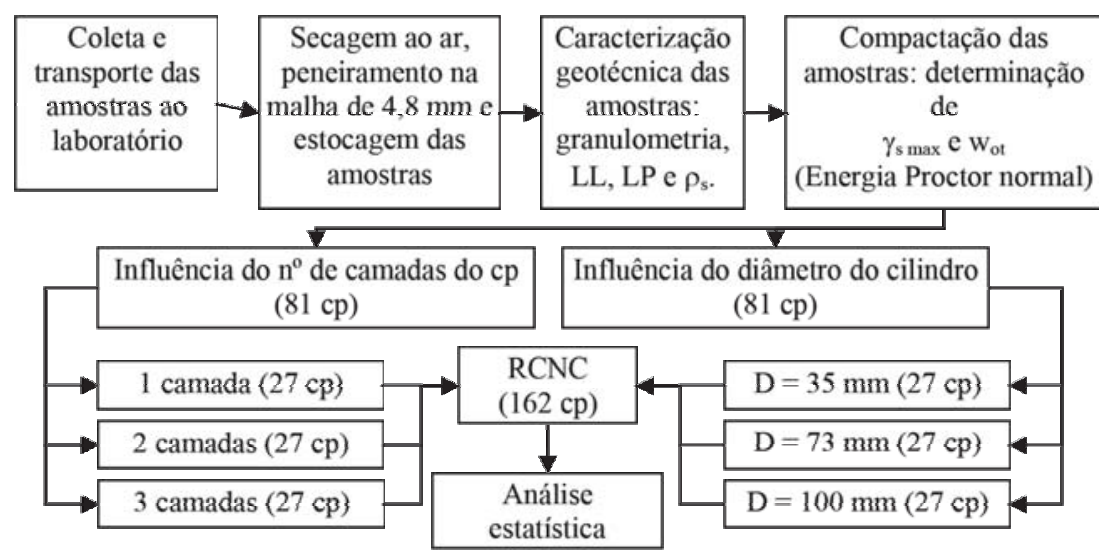

Obs.: cp corpo de prova; cp 9 corpos de prova para cada teor de umidade: $\mathrm{w}_{\mathrm{ot}}-3 \% \mathrm{w}_{\mathrm{ot}} \mathrm{e} \mathrm{w}_{\mathrm{ot}}+2 \%$; e RCNC '! resistência à compressão não confinada.

Figura 1 - Quadro-resumo do programa de ensaios de cada um dos solos analisados.

Figure 1 - Layout of the testing program for each of the tested soils.

Tabela 1 - Resultados dos ensaios de caracterização geotécnica e compactação das amostras dos solos 1 e 2

Table 1 - Geotechnical characterization and compaction data of soil samples 1 and 2

\begin{tabular}{|c|c|c|c|}
\hline \multicolumn{2}{|c|}{ Resultados de ensaios de caracterização geotécnica e compactação } & \multicolumn{2}{|c|}{ Solos } \\
\hline 1 & 2 & & \\
\hline Limite de liquidez: $w_{\mathrm{L}}(\%)$ & 74 & NL & \\
\hline Índice de plasticidade: IP (\%) & 28 & NP & \\
\hline \multirow[t]{2}{*}{ Massa específica dos grãos: $\mathrm{r}_{\mathrm{s}}\left(\mathrm{g} / \mathrm{cm}^{3}\right)$} & 2,990 & 2,780 & \\
\hline & Argila (f $0,002 \mathrm{~mm}$ & 66 & 7 \\
\hline Granulometria & Silte $(0,002<\mathrm{f} \quad 0,06 \mathrm{~mm})$ & 4 & 25 \\
\hline \multirow[t]{2}{*}{$(\% \text { em pesoque passa })^{*}$} & Areia $(0,06<\mathrm{f} \quad 2 \mathrm{~mm})$ & 30 & 68 \\
\hline & Pedregulho ( $\mathrm{f}>2 \mathrm{~mm}$ ) & 0 & 0 \\
\hline Massa específica aparente seca máxima, $\gamma_{\mathrm{s}}\left(\mathrm{g} / \mathrm{cm}^{3}\right)$ & & 1,418 & 1,742 \\
\hline Umidade ótima, $w_{\text {ot }}(\%)$ & & 30,50 & 14,90 \\
\hline
\end{tabular}

"Classificação granulométrica segundo a NBR 6502 (ABNT, 1995).

*Granulometric classification according to NBR 6502 (ABNT, 1995).

os solos 1 e 2 são classificados, respectivamente, nos grupos A-7-5 (18) e A-2-4 (0), segundo o Sistema de Classificação do Transportation Research Board (TRB) dos Estados Unidos (DNIT, 2006). A seguir, apresentamse as discussões relativas às análises da influência do número de camadas e do diâmetro do corpo de prova na massa específica aparente seca $(\gamma)$ e na resistência à compressão não confinada (RCNC).

\subsection{Análise da influência do número de camadas do corpo de prova}

Para fins de análise da influência do número de camadas dos corpos de prova na curva de compactação e na resistência mecânica dos solos, adotou-se como referência a compactação em três camadas, sendo esse o procedimento mais utilizado nos ensaios de compactação no Brasil.

Como referido anteriormente, na Figura 2 (itens a e b) são apresentados os resultados encontrados nos parâmetro $\gamma_{\mathrm{s}}$ e RCNC dos solos 1 e 2. Observase que, tanto os valores de $\tilde{\mathrm{a}}_{\mathrm{s}}$ quanto de RCNC, foram menores quando os solos foram compactados em uma camada, sendo esse comportamento mais evidente no solo 2. No entanto, a compactação em duas camadas apresentou resultados que se aproximaram da compactação em três camadas.

Nota-se também, nessa figura, que as diferenças entre médias mostradas na Figura 3 (itens a e b) referentes ao parâmetro $\gamma_{\mathrm{s}}$ foram pequenas para fins práticos de

Revista Árvore, Viçosa-MG, v.39, n.3, p.535-542, 2015 

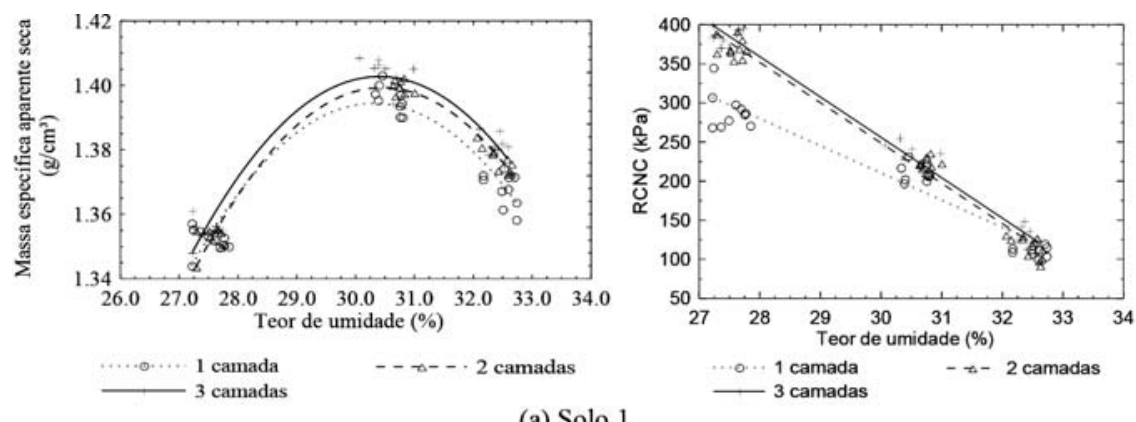

(a) Solo 1
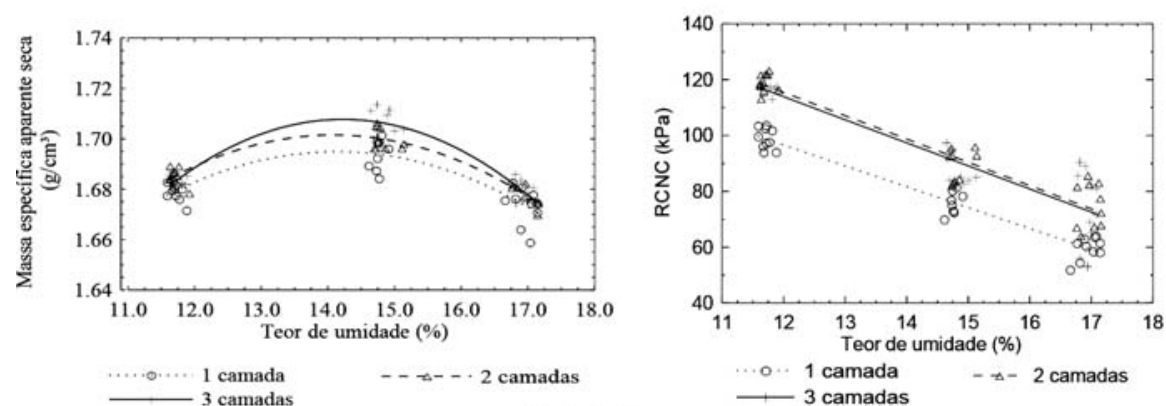

(b) Solo 2

Estudo da influência do número de camadas do corpo de prova: resultados de nove repetições
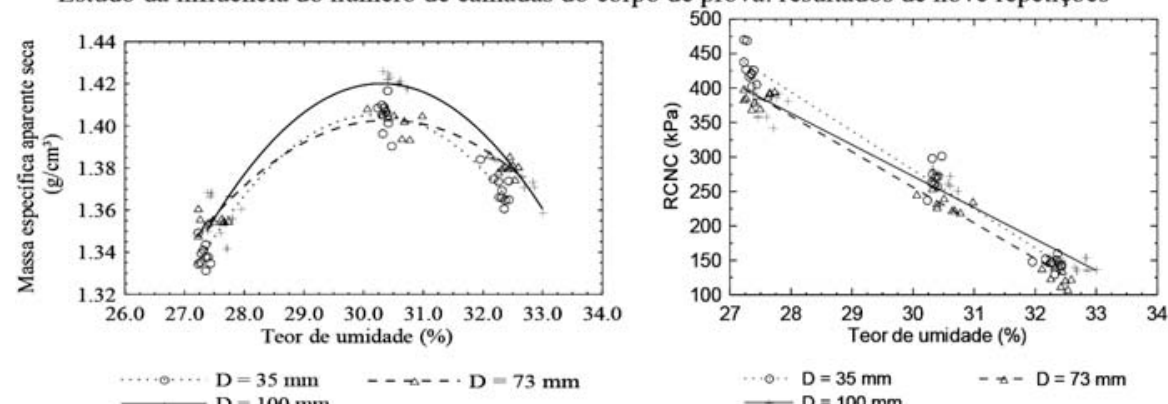

(c) Solo 3
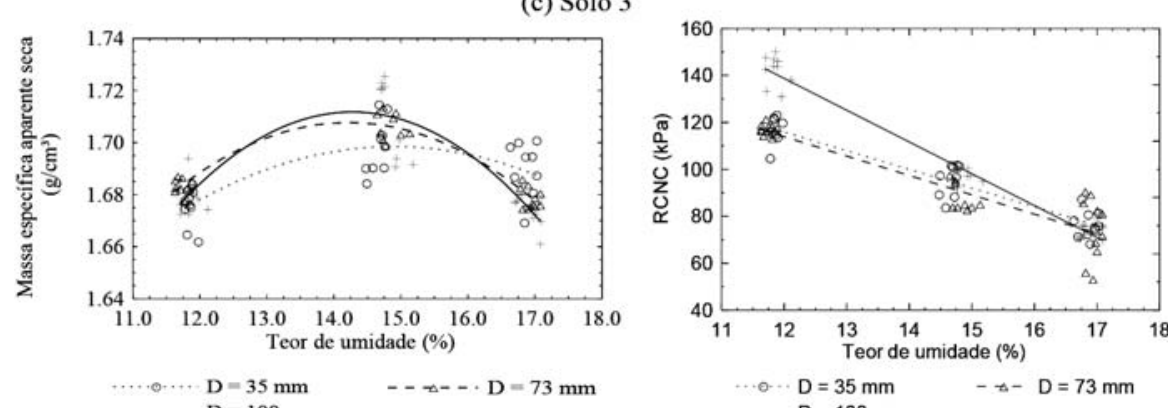

(c) Solo 3

Estudo da influência do diâmetro do corpo de prova: resultados de nove repetições

Figura 2 - Curvas de compactação e resistência à compressão não confinada dos solos 1 e 2: influência do número de camadas $(\mathrm{a}, \mathrm{b})$ e diâmetro $(\mathrm{c}, \mathrm{d})$ do corpo de prova.

Figure 2 - Curves of compaction and unconfined compression strength of soils 1 and 2: influence of number of layers $(a, b)$ and diameter $(c, d)$ of the specimen. 


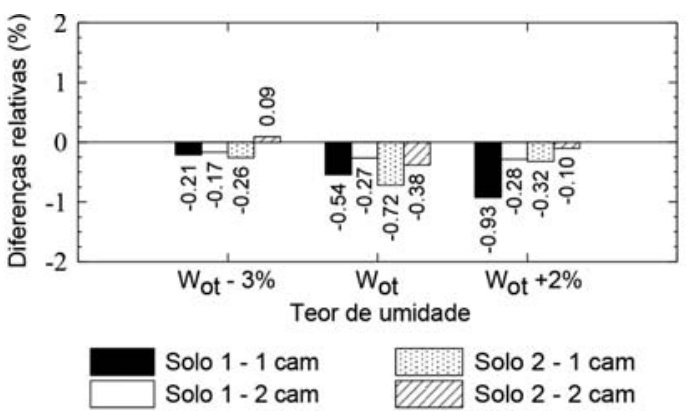

(a) $\gamma_{\mathrm{s}}$

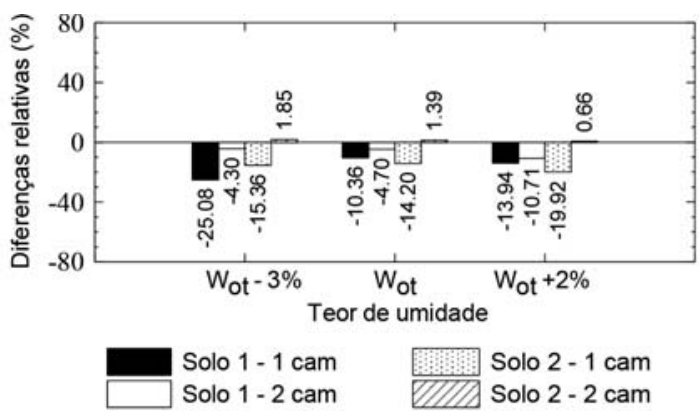

(b) RCNC

Estudo da influência do número de camadas do corpo de prova

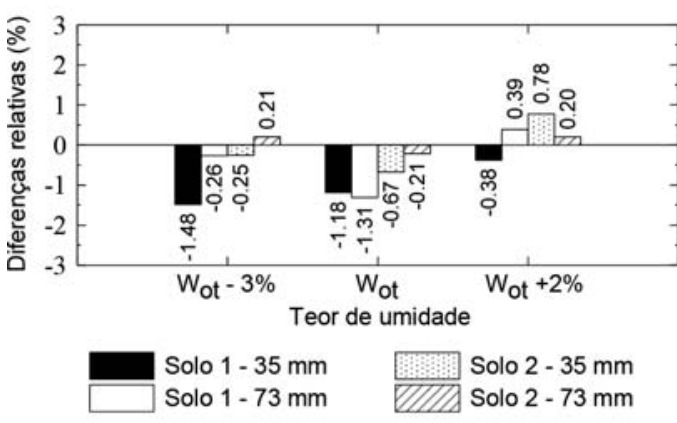

(c) $\gamma_{\mathrm{s}}$

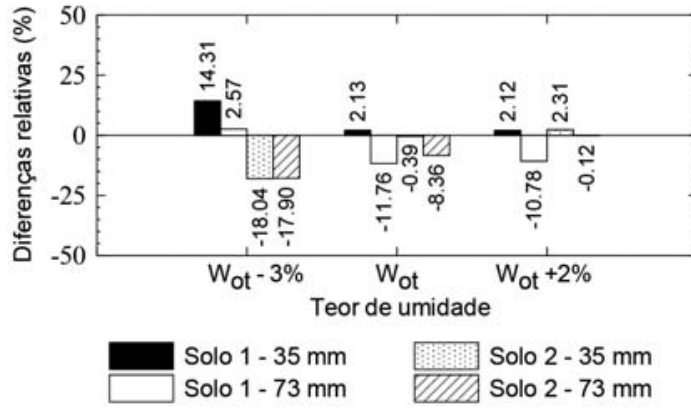

(d) $\mathrm{RCNC}$

Estudo da influência do diâmetro do corpo de prova

Figura 3 - Diferenças relativas entre médias dos parâmetros $\tilde{a}_{d}$ e RCNC das amostras dos solos 1 e 2, considerando-se como referência a compactação em três camadas (a, b) e o diâmetro de $100 \mathrm{~mm}(\mathrm{c}, \mathrm{d})$.

Figure 3 - Relative differences between the $\tilde{a}_{d}$ and RCNC average parameters of soil samples 1 and 2, regarding the three layers compaction $(a, b)$ and the $100 \mathrm{~mm}$ diameter as references.

Tabela 2 - Resultados da análise de variância aplicada aos resultados do parâmetro RCNC dos solos 1 e 2, considerandose os estudos da influência do número de camadas e do diâmetro do corpo de prova.

Table 2 - Results of the variance analysis applied to the RCNC parameter of soils 1 and 2 regarding the analysis of the influence of the number of layers and diameter of the specimen.

\begin{tabular}{|c|c|c|c|c|c|c|}
\hline \multicolumn{7}{|c|}{ Estudo da influência do número de camadas do corpo de prova } \\
\hline Relações de número de camadas & & C do & & & C do & \\
\hline $\begin{array}{c}1 \text { camada versus } \\
3 \text { camadas }\end{array}$ & $\begin{array}{c}\mathrm{W}_{\text {ot }}-3 \% \\
*\end{array}$ & $\begin{array}{c}\mathrm{W}_{\text {ot }} \\
*\end{array}$ & $\begin{array}{c}\mathrm{W}_{\text {ot }}+2 \% \\
*\end{array}$ & $\mathrm{~W}_{\text {ot }}-3 \%$ & $\mathrm{~W}_{*}$ & $\begin{array}{c}\mathrm{W}_{\text {ot }}+2 \% \\
*\end{array}$ \\
\hline $\begin{array}{c}2 \text { camadas versus } \\
3 \text { camadas }\end{array}$ & ns & $\mathrm{ns}$ & ns & ns & ns & ns \\
\hline \multicolumn{7}{|c|}{ Estudo da influência do diâmetro do corpo de prova } \\
\hline Relações de diâmetros & \multicolumn{3}{|c|}{ RCNC do solo 1} & \multicolumn{3}{|c|}{ RCNC do solo 2} \\
\hline $35 \mathrm{~mm}$ versus $100 \mathrm{~mm}$ & $\begin{array}{c}\mathrm{W}_{\text {ot }}-3 \% \\
*\end{array}$ & $\begin{array}{l}\mathrm{W}_{\text {ot }} \\
\mathrm{ns}\end{array}$ & $\begin{array}{c}\mathrm{W}_{\text {ot }}+2 \% \\
\mathrm{~ns}\end{array}$ & $\mathrm{~W}_{\text {ot }}-3 \%$ & $\begin{array}{l}\mathrm{W}_{\text {ot }} \\
\mathrm{ns}\end{array}$ & $\begin{array}{c}\mathrm{W}_{\mathrm{ot}}+2 \% \\
\mathrm{~ns}\end{array}$ \\
\hline $73 \mathrm{~mm}$ versus $100 \mathrm{~mm}$ & ns & $*$ & $*$ & $*$ & $\mathrm{~ns}$ & ns \\
\hline
\end{tabular}

(*) Indica a ocorrência de diferenças estatisticamente significativas e (ns) indica que não existem diferenças estatisticamente significativas a $5 \%$ de probabilidade.

Revista Árvore, Viçosa-MG, v.39, n.3, p.535-542, 2015 
construção de estradas, não chegando a $1 \%$, mesmo para a compactação em uma camada. No entanto, quanto ao parâmetro RCNC, as diferenças entre as resistências médias foram expressivas, chegando a $11 \%$ para a compactação em duas camadas e a $25 \%$ para a compactação em uma camada.

A menor resistência encontrada nos corpos de prova compactados em uma camada se deveu, provavelmente, à heterogeneidade do grau de compactação ao longo da altura desses corpos. Devido ao atrito das partículas, com as paredes do cilindro de compactação e entre si, parte da energia de compactação pode ter sido absorvida pela parcela superior do corpo de prova, produzindo-se, então, grau de compactação maior no seu topo e menor na sua base. Esse efeito foi mais evidente no solo 2, granular, com $68 \%$ de fração areia. Entretanto, quanto ao solo 1, o efeito do atrito foi menor, pois se trata de um solo argiloso com $66 \%$ de fração argila, e o comportamento observado o aproximou daquele da compactação em duas e em três camadas, exceto no teor de umidade $\mathrm{w}_{\text {ot }}-3 \%$, podendo associar-se tal comportamento a uma possível influência da sução no ramo seco da curva de compactação.

\subsection{Análise da influência do diâmetro do cilindro de compactação}

$\mathrm{Na}$ Figura 2 (itens b e c), apresentam-se os resultados do estudo da influência do diâmetro do cilindro na curva de compactação e na resistência mecânica dos solos 1 e 2, considerando corpos de prova compactados estaticamente com diâmetros de $35 \mathrm{~mm}, 73 \mathrm{~mm}$ e 100 $\mathrm{mm}$. Com base nos resultados apresentados, observouse, em geral, que o comportamento quanto ao parâmetro RCNC dos corpos de prova compactados no diâmetro de $100 \mathrm{~mm}$ diferiu dos demais, notadamente no solo 2 , no teor de umidade $\mathrm{w}_{\text {ot }}-3 \%$.

Na Figura 3 (itens c e d), apresentam-se as diferenças relativas entre os valores médios de $\mathrm{g}_{\mathrm{s}}$ e RCNC obtidos, tomando como referência (linha de diferenças relativas igual a zero) os resultados de corpos de prova compactados no diâmetro de $100 \mathrm{~mm}$. Observa-se, nessa figura, que o comportamento do parâmetro $g_{\mathrm{s}}$ é relativamente uniforme, uma vez que, independentemente do tipo de solo, as diferenças encontradas foram pequenas para fins práticos de construção de estradas, chegando ao máximo de 1,48\%. Entretanto, as diferenças determinadas no parâmetro RCNC foram mais significativas, atingindo o valor de $14,31 \%$ no solo 1 e de $18,04 \%$ no solo 2 , no teor de umidade $\mathrm{w}_{\text {ot }}-3 \%$. Explica-se a ocorrência de maior variabilidade no parâmetro RCNC, pois, contrariamente ao parâmetro $\mathrm{g}_{\mathrm{s}}$, que se relaciona com o arranjo espacial das partículas, este é influenciado pela estrutura do solo, entendida como a conjugação do arranjo espacial de suas partículas, composição e forças interpartículas, como destacou Mitchell (1993).

\subsection{Estudos de análise de variância}

A fim de verificar a validade estatística das análises apresentadas, aplicaram-se um teste estatístico e a análise de variância a 5\% de probabilidade ao parâmetro RCNC dos solos 1 e 2, considerando os estudos de influência do número de camadas e do diâmetro do corpo de prova, cujos dados se encontram na Tabela 2. Com base nos resultados, tem-se que: (ii) confirmou-se o que foi discutido no subitem (4.1), ou seja, as diferenças entre as compactações realizadas em uma e em três camadas foram estatisticamente significativas, e as diferenças entre as compactações em duas e três camadas não foram estatisticamente significativas a 5\% de probabilidade; e (ii) pode-se afirmar que houve diferenças estatisticamente significativas entre a compactação com o cilindro de diâmetro de $100 \mathrm{~mm}$ e os demais a $5 \%$ de probabilidade, como se segue: (a) quanto ao solo 1 , nos três teores de umidade analisados; e (b) quanto ao solo 2, apenas no teor de umidade $\mathrm{W}_{\text {ot }}-3 \%$.

\section{CONCLUSÃO}

Com base nos resultados e considerando os parâmetros g e RCNC, pode-se concluir que: (i) quanto à influência do número de camadas do corpo de prova, foi observado que houve diferenças significativas entre as compactações realizadas em uma e em três camadas, notadamente no solo 2, arenoso; no entanto, não foram encontradas diferenças importantes entre as compactações em duas e três camadas, podendo-se afirmar que a compactação em duas camadas leva a resultados semelhantes à compactação em três camadas, nos solos analisados; e (ii), quanto ao efeito do diâmetro do cilindro de compactação, notaram-se diferenças significativas entre a compactação no diâmetro de $100 \mathrm{~mm}$ e os demais (diâmetros de $73 \mathrm{~mm}$ e $35 \mathrm{~mm}$ ), no solo 1, argiloso, e no solo 2 esse fato ocorreu apenas no ramo seco da curva de compactação.

Revista Árvore, Viçosa-MG, v.39, n.3, p.535-542, 2015 


\section{AGRADECIMENTOS}

À Fundação de Amparo à Pesquisa do Estado de Minas Gerais (FAPEMIG), pelo apoio financeiro (Processos TEC-APQ-00378-08 e TEC PPM 00081-10); à Coordenação de Aperfeiçoamento de Pessoal de Nível Superior (CAPES) e ao Conselho Nacional de Desenvolvimento Científico e Tecnológico (CNPq), pela concessão de bolsa de estudo ao primeiro autor e de iniciação científica ao último autor; e à Universidade Federal de Viçosa, pela infraestrutura disponibilizada para a execução dos trabalhos.

\section{REFERÊNCIAS}

\section{ASSOCIAÇÃO BRASILEIRA DE NORMAS} TÉCNICAS - ABNT. NBR 7181: Solo - análise granulométrica. Rio de Janeiro: 1984a. 13p.

\section{ASSOCIAÇÃO BRASILEIRA DE NORMAS} TÉCNICAS - ABNT. NBR 6459: Solo determinação do limite de liquidez. Rio de Janeiro: 1984b. 6p.

\section{ASSOCIAÇÃO BRASILEIRA DE NORMAS TÉCNICAS - ABNT. NBR 7180: Solo - determinação do limite de plasticidade. Rio de Janeiro: 1984c. 3p.}

\section{ASSOCIAÇÃO BRASILEIRA DE NORMAS} TÉCNICAS - ABNT. NBR 6508: Grãos de solos que passam na peneira de $4,8 \mathrm{~mm}$ - determinação da massa específica. Rio de Janeiro: 1984d. 8p.

\section{ASSOCIAÇÃO BRASILEIRA DE NORMAS} TÉCNICAS - ABNT. NBR 7182: Solo - ensaio de compactação. Rio de Janeiro: 1986. 10p.

\section{ASSOCIAÇÃO BRASILEIRA DE NORMAS} TÉCNICAS - ABNT. NBR 12770: Solo coesivo - determinação da resistência à compressão não confinada. Rio de Janeiro: 1992. 4p.

\section{ASSOCIAÇÃO BRASILEIRA DE NORMAS} TÉCNICAS - ABNT. NBR 6502: Solos e rochas. Rio de Janeiro: 1995. 18p.

CRISPIM, F.A. Influência de variáveis de compactação na estrutura dos solos: caracterizações geotécnica, química, mineralógica e microestrutural. 2010. 125f. Tese (Doutorado em Engenharia Civil) - Universidade Federal de Viçosa, Viçosa, MG, 2010.

Revista Árvore, Viçosa-MG, v.39, n.3, p.535-542, 2015
CRISPIM, F.A.; LIMA, D.C.; SCHAEFER, C.E.G.R.; SILVA, C.H.C.; CARVALHO, C.A. B.; BARBOSA, P.S.A.; BRANDÃO, E.H. The influence of laboratory compaction methods on soil structure: mechanical and micromorphological analysis. Soils and Rocks, v.34, n.1, p.91-98, 2011.

CRISPIM, F.A.; LIMA, D.C.; SILVA, C.H.C.; SCHAEFER, C.E.G.R.; CARVALHO, C.A.B.; BARBOSA, P.S.A.; STEHLING, R.S. Influência de métodos de compactação em laboratório na permeabilidade de um solo residual de gnaisse. In: CONGRESSO BRASILEIRO DE MECÂNICA DOS SOLOS E ENGENHARIA GEOTÉCNICA, 16 COBRAMSEG 2012, Porto de Galinhas, Pernambuco, 2012. Anais... 2012. 6p.

DEPARTAMENTO NACIONAL DE ESTRADAS DE RODAGEM - DNER. ME 228/94: Solos compactação em equipamento miniatura. Rio de Janeiro: 1994. 14p.

\section{DEPARTAMENTO NACIONAL DE}

INFRAESTRUTURA DE TRANSPORTES-DNIT Manual de pavimentação. Rio de Janeiro: 2006. 274p.

MACHADO, C.C.; SILVA, E. Planejamento de estradas florestais ambientalmente corretas. In: SIMPÓSIO BRASILEIRO DE COLHEITAE TRANSPORTE FLORESTAL, 5, Porto Seguro, Bahia, 2001. Anais... 2001. p.28-44.

MACHADO, C.C.; SANTANNA, G.L.; LIMA, D.C.; CARVALHO, C.A.; PEREIRA, R.S.; FERNANDES, D.C.M. Comportamento geotécnico de misturas granulométricas de solo-grits. Revista Árvore, v.33, n.3, p.555-562, 2009.

MITCHELL, J.K. Fundamentals of soil behavior. New York: USA: John Wiley, 1993. 437p.

NOGAMI, J.S.; VILLIBOR, D.F. Pavimentação de baixo custo com solos lateríticos. São Paulo: Villibor, 1995. 213p.

SILVA, T.O.; CARVALHO, C.A.B.; LIMA, D.C.; CALIJURI, M.L.; MACHADO, C.C.; OLIVEIRA, T.M. Avaliação do subleito de rodovias vicinais de baixo volume de tráfego por meio de ensaios geotécnicos. Revista Árvore, v.35, n.4, p.825-833, 2011. 